\title{
Finite element modelling of the effective elastic properties of partially saturated rocks
}

\author{
Dina Makarynska ${ }^{1 *}$, Boris Gurevich ${ }^{1,2}, \operatorname{Radim~Ciz}^{2}$, Christoph H. Arns $^{3}$ and Mark A. \\ Knackstedt $^{3}$
}

${ }^{1)}$ Curtin University of Technology, Department of Exploration Geophysics, GPO Box U1987, Perth, Western Australia 6845. E-mail: Dina.Makarynska@geophy.curtin.edu.au; Boris.Gurevich@geophy.curtin.edu.au

2) CSIRO Petroleum, ARRC, 26 Dick Perry Ave, Kensington, Perth, WA 6151, Australia, E-mail: Radim.Ciz@csiro.au

3) Australian National University, Department of Applied Mathematics, Research School of Physical Sciences and Engineering, Canberra ACT 0200, Australia. E-mail: Christoph.Arns@anu.edu.au; Mark.Knackstedt@anu.edu.au

*Corresponding author. Postal address: Curtin University of Technology, Department of Exploration Geophysics, GPO Box U1987, Perth, Western Australia 6845.

Tel: (+61 8) 9266-3521; Fax: (+61 8) 9266-3407 


\begin{abstract}
Simulation of effective physical properties from microtomographic 3D images of porous structures allows one to relate properties of rocks directly to their microstructure. A static FEM code has been previously used to estimate effective elastic properties of fully saturated monomineralic (quartz) rock under wet and dry conditions. We use the code to calculate elastic properties under partially saturated conditions. The numerical predictions are compared to the Gassmann theory combined with Wood's formula (GW) for a mixture of pore fluids, which is exact for a monomineralic macroscopically homogeneous porous medium.

Results of the numerical simulations performed for two Boolean sphere pack distributions show significant deviation from the GW limit and depend on the spatial distribution of fluids. This is shown to be a numerical artefact caused by incomplete equilibration of fluid pressure, which is primarily due to insufficient spatial resolution.

To investigate the effect of pore-size and pore geometry, we perform FEM simulations for a model with regular pore geometry, where all pore channels have the same size and shape. Accuracy of these simulations increases with the total cross-section area of the channels and the size of individual channels. For the case where the total cross-section of the channels is large enough (on the same order as total porosity), there is a minimum of 4 voxels per channel diameter required for adequate fluid pressure equilibration throughout the pore space. Increasing the spatial resolution of the digital models reduces the discrepancy between the simulations and theory, but unfortunately increases the memory and CPU requirements of the simulations.
\end{abstract}

Keywords: Poroelasticity; Partial saturation; Effective elastic properties of rocks; Finite Element Method; Gassmann theory 


\section{Introduction}

Quantitative interpretation of geophysical data requires the knowledge of relationships between physical properties of rocks and their microstructure. With the advances in computer technology, it has become feasible to compute macroscopic physical properties of porous rocks from its microstructural information using rigorous numerical simulations of physical experiments in realistic pore space geometries. This approach, which has become known as digital (or computational) rock physics, has been used to model the effect of pores, fractures and fluids on the effective acoustic properties (Arns et al., 2002; Grechka, 2003; Gurevich et al., 2005; Roberts and Garboczi, 2000; Saenger et al., 2004;) as well as geometrical, hydraulic and electric properties of rocks (Arns et al., 2001, 2004; Auzerais et al., 1996; Keehm et. al., 2004; Schwartz et al., 1994; Spanne et al., 1994). The computational rock physics technology relies on digitalized microstructural information which can be obtained either through x-ray microtomography (Dunsmuir et al., 1991; Flannery et al., 1987; Spanne et al., 1994), laser confocal microscopy (Fredrich et al., 1995), or stochastic models (Adler et al., 1992; Arns, 2002; Roberts and Garboczi, 2002; Yeong and Torquato, 1998). The numerical approach allows one to study properties of complex multi-phase materials with physically realistic phase distributions. However, computer simulations are not exact and their accuracy is limited by the simulation algorithm and by computational parameters such as spatial discretization. Therefore, in order to optimize the computational parameters, it is important to test the results of such simulations against known rigorous solutions.

One algorithm that has proved effective in simulations of the linear elastic properties of rocks is a finite-element method or FEM (Arns et al., 2002; Garboczi, 1998). There is a difficulty in testing such algorithms as values of the elastic properties are not known exactly for a porous material of any particular 3-D pore geometry. However, one rigorous theory applicable to the elastic properties in such a case is the Gassmann theory (1951), which is exact for a 
monomineralic macroscopically homogeneous porous medium with fully connected statistically isotropic pore space and no chemical/physical interaction between rock and pore fluid. In this paper, we propose a test scenario for static numerical simulations for a medium saturated with a fluid mixture. This test scenario is based on application of the Gassmann theory. When the Gassmann theory is applied to a medium saturated with a fluid mixture, the effective bulk modulus of a mixture is computed with Wood's equation (1955), which is an exact isostress formula for a mixture of Newtonian fluids. Wood's equation implies that fluid pressure is spatially uniform throughout the pore space. Since all the assumptions of the Gassmann theory could be simulated by FEM, a comparison of the numerically predicted parameters with the theory provides a natural test of the accuracy of numerical results. Arns et al. (2002) have shown that FEM simulations on media saturated with one fluid agree with the Gassmann predictions for a variety of porous structures. Here we extend those simulations to porous rocks saturated by a mixture of two fluids and test the predictions against Gassmann solutions. The goal is to determine computational parameters that ensure accurate and robust simulations.

The paper is organized as follows. Section 2 gives a brief outline of FEM used to derive the linear elastic properties. Section 3 describes the test scenario of the accuracy of the numerical simulations. Section 4 deals with numerical predictions of the effective elastic moduli. First, we perform the numerical experiments for Boolean random models of quartz saturated with gas and water. Then Wood's formula is verified for models of a fluid mixture (no solid phase). In the next set of numerical experiments, we focus on models of partially saturated idealized materials with regular geometries. We investigate the effect of pore channel size and the total area of water-gas interface on numerical predictions. Finally, we examine the effect of spatial resolution of the images on the FEM code performance. Section 5 summarizes our conclusions. 


\section{Numerical method}

A method particularly suitable for the prediction of linear effective elastic properties of porous rocks from its microstructure is FEM by Garboczi, 1998, Arns et al., 2002. FEM is specially designed to handle arbitrary voxel-based models. Given bulk and shear moduli of material solid constituents and fluid phases, FEM can simulate the macroscopic physical properties, such as the effective elastic modulus, on 3D images of porous rocks at the pore scale. The method uses a variational formulation of the static linear elastic equations, and finds the solution by minimising the elastic energy using a fast conjugate-gradient solver. A macroscopic strain is applied, with the resultant average stress or the average elastic energy giving the effective elastic moduli. The images are assumed to have periodic constant-strain boundary conditions.

FEM discretizes digital images that each voxel in 3-D is taken to be a 3-linear finite element. In practice, the accuracy of the results is limited by discretization errors (how well a digital model can be resolved) (Roberts and Garboczi, 2000). However, the number of voxels depends on computer memory and speed (230 bytes for a voxel). Since the numerical simulations are computationally expensive, there has clearly been a trade-off between number of voxels and computational speed. In this work, we use digital images at a scale from (180) ${ }^{3}$ up to $(400)^{3}$ voxels to investigate the effect of spatial resolution on the accuracy of the FEM predictions. 


\section{Numerical test scenario}

To examine the accuracy of FEM simulations we apply them to a rock saturated with a mixture of two fluids. Because FEM predicts static effective properties, they can be compared with the exact solution based on the Gassmann theory.

Gassmann’s equation (Gassmann, 1951) for an effective bulk modulus of a fluidsaturated porous material can be written in the form

$$
K=K_{0}+\sigma^{2} M
$$

where

$$
M=\left[\frac{\sigma-\phi}{K_{g}}+\frac{\phi}{K_{f}}\right]^{-1},
$$

is called the pore space modulus and

$$
\sigma=1-\frac{K_{0}}{K_{g}},
$$

is Biot-Willis effective stress coefficient, $\phi$ is porosity, $K_{0}, K_{g}$, and $K_{f}$ are bulk moduli of the dry frame, solid grain material and fluid, respectively.

If the pore space is filled with a mixture of $n$ fluids, the bulk modulus $K_{f}$ of the fluid mixture is given exactly by Wood's formula (Wood, 1955)

$$
\frac{1}{K_{f}}=\sum_{i=1}^{n} \frac{x_{i}}{K_{i}},
$$

where $x_{i}$ and $K_{i}$ are the volume fractions and bulk moduli of the individual fluid phases, respectively. Wood's formula is often called the isostress average because it gives the ratio of stress to average strain when all phases are assumed to have the same stress (pressure). This situation occurs when the phases are mixed at the finest scales, and pore pressure increments of 
each fluid equilibrate with each other to a single average value. The collection of phases in such case could be replaced with a single "effective fluid".

Specifically, for a mixture of two fluids, say, gas and water, Eq. (4) reads

$$
\frac{1}{K_{f}}=\frac{S_{w}}{K_{w}}+\frac{1-S_{w}}{K_{g a s}},
$$

where $S_{w}$ is water saturation, $K_{w}$ is the bulk modulus of water and $K_{\text {gas }}$ is the bulk modulus of gas. Substitution of the fluid modulus as given by Eq. (5) into Gassmann's Eq. (1) yields socalled Gassmann-Wood (GW) formula (Johnson, 2001; White, 1983)

$$
K_{G W}=K_{0}+\sigma^{2}\left[\frac{\sigma-\phi}{K_{g}}+\frac{\phi \cdot S_{w}}{K_{w}}+\frac{\phi \cdot\left(1-S_{w}\right)}{K_{g a s}}\right]^{-1} .
$$

Eq. (6) gives the static elastic modulus $K_{G W}$ of a rock, where all fluid phases in the pore space are immiscible, and there are no hydraulically isolated pores. This is the situation easily simulated by FEM or other static algorithm, and therefore comparison of the simulation results with the predictions of Eq. (6) gives a measure of validity of the simulations.

To check how well the simulations predict this particular limit, we also compare them with the high-frequency limit. In the high-frequency limit, the fluid pressure does not have time to equilibrate, and regions of the porous medium saturated with different fluids behave as if they were hydraulically isolated (Bear, 1988; Dvorkin et al., 1999; Johnson, 2001; Toms et al., 2006). The higher-frequency limit of the elastic bulk modulus is given by Hill’s (1961) average:

$$
K_{G H}=\left[\sum_{i=1}^{n} \frac{x_{i}}{K_{i}+4 / 3 \mu}\right]^{-1}-4 / 3 \mu,
$$


where $K_{G H}$ is the effective bulk modulus of the rock, $n$ is the number of fluid phases, $x_{i}$ is the volume fraction of the $i$ th fluid, $\mu$ is the shear modulus of the rock, and $K_{i}$ is the bulk modulus of the rock completely saturated with $i$ th fluid. Specifically, for two fluids, water and gas, we have:

$$
K_{G H}=\left[\frac{S_{w}}{K_{w}^{\text {sat }}+4 / 3 \mu}+\frac{1-S_{w}}{K_{\text {gas }}^{\text {sat }}+4 / 3 \mu}\right]^{-1}-4 / 3 \mu,
$$

where saturated moduli $K_{w}^{\text {sat }}$ and $K_{\text {gas }}^{\text {sat }}$ are given by Gassmann's Eq. (1) - (3) with the fluid modulus $K_{f}$ equal to $K_{w}$ and $K_{\text {gas }}$. The modulus given by Eq. (8) is known as the GassmannHill or GH limit (Johnson, 2001).

The difference between GW and GH limits yields the magnitude of frequency dispersion of the bulk modulus of a fluid-saturated rock. In the numerical experiments that follow, we compare any deviation of the numerical results from the GW limit against this difference.

\section{Numerical experiments}

\subsection{FEM simulation of effective elastic properties for Boolean random models}

We use two types of three-dimensional $(180 \times 180 \times 180)$ digital models of partially saturated rocks (Fig. 1) to predict their linear effective elastic properties employing FEM. The model morphologies were generated using a simple Boolean overlapping spheres method (Arns, 2002; Knackstedt et al., 2003) for various degrees of saturation ranging from dry to fully saturated conditions with porosity approximately equal to 0.25 . The Boolean models often used to represent irregular spatial structures generated by gradual build up of a phase via the overlap of permeable particles such as spheres, each with arbitrary location and orientation. 
Figure 1.

We consider two models with different distribution of fluid patches. The locally patchy model (Fig. 1 a) has uniformly distributed fluid patches, whereas macro-patchy model (Fig. 1 b) contains a single spherical inclusion in the middle of the porous system.

Three-dimensional digitized model or image is a collection of discrete voxels in which each voxel can, in principle, be a different phase of material. We use images in a binary form with " 1 " and " 3 " assigned to the voxels that fall into the fully connected pore space and represent gas and water accordingly, "2" assigned to the voxels standing for the solid phase (quartz). The elastic properties for quartz skeleton are taken to be $K_{g}=37 \mathrm{GPa}, \mu=44 \mathrm{GPa}$ (Mavko et al., 1998). Water is modelled at $T=200^{\circ} \mathrm{C}$ and $40 \mathrm{MPa}$ pressure with $K_{w}=2.22 \mathrm{GPa}, \mu=0 \mathrm{GPa}$, and gas with $K_{\text {gas }}=0.05 \mathrm{GPa}, \mu=0 \mathrm{GPa}$. FEM allows to model fluids as solids with zero shear modulus. To ensure full equilibrium of fluid flow pore fluids are treated by FEM as frictionless with zero viscosity.

Figure 2.

The results of numerical simulations performed for the Boolean models are demonstrated in Fig. 2. The Gassmann theory described above stipulates that the effective elastic properties of rocks represented by such models should be independent of the geometry and size of fluid patches. Hence, the predicted effective elastic moduli should be the same for both models and agree with the GW theory. However, the numerical estimates show significant differences between the fluid models on, and both disagree with the GW limit.

Figure 3. 
In Fig. 3, we output the central slices illustrating the pressure field at the end of the relaxation process for both models. According to Pascal's law, when fluid is at rest in the absence of body forces the pressure is the same throughout all fluid points. However, we can observe significant variation in the pressure field over the pore space.

Figure 4.

To investigate this discrepancy with the theory, we verify Wood's formula by testing the FEM code on models with two free fluid phases (water and gas) without a solid phase. The models geometries are shown in Fig. 4. The first model contains a single spherical gas inclusion in the centre of a liquid cube, while the second one has several randomly distributed gas bubbles of different size. Wood's formula uses a concept of a compressible homogeneous pore fluid to treat a fluid mixture, each component of which is assumed to have the same stress. Hence, the numerical results should be the same for both models regardless of their geometries. A comparison of the predicted moduli for different levels of saturation (0.7-1) in Fig. 5 demonstrates excellent agreement of both models with Wood's formula. This proves the capability of the FEM code to predict the effective elastic moduli of a fluid mixture.

Figure 5.

These results suggest that in the presence of solid particles the FEM simulations fail to equilibrate fluid pressure throughout the pore space, even if though the pore space is fully interconnected. One possible explanation for this is an insufficient number of finite elements across thin fluid channels (i.e., insufficient density of FEM grid in channels). It is difficult to explore this effect using random packs of spheres (or any other random model) as all pores and 
pore channels have different sizes. Therefore below we investigate this effect using models with regular pore and channel geometry.

\subsection{Effect of pore channel size}

To explore the extent to which small (in voxels) pore connections can be resolved properly by the FEM code, we conduct numerical experiments on the images of the idealized partially saturated rocks with regular pore geometry. In contrast to random Boolean models with variable size of interconnections between pores, we generate a simple three dimensional model of poroelastic material (200x200x200), where a cubic inclusion ('pore') in the centre of a solid (quartz) cube is connected with other 'pores' by channels of adjustable cross-section (Fig. 6).

Figure 6.

To test pressure equilibration between liquid and gas (the 'worst' case), we assume that the inner pore is filled with gas while the outer pores are water-filled, the gas/water interface crosses the channels. Since the fluid-fluid interface separates the fluids within the channels, we could control the area of the interface by changing the cross-sectional area of the channels. The numerical simulations are carried out for the images with 0.25 porosity considering three levels of saturation: dry, 0.75 , and fully saturated.

Figure 7.

In Fig. 7, we compare the results of three sets of experiments with different crosssectional area of the channels, ranging from 4 elements (voxels) to 900. For the model with the widest channels and, consequently, the largest total contact area between the fluids, the FEM 
code yields results which are in excellent agreement with the GW limit, demonstrating that the pressure field is fully equilibrated. In contrast, the predicted effective moduli for the image with the thinnest channels are close to the GH limit. We observe significant pressure variation over the pore space with extreme values in the channels. For the model with cross-sectional area of 100 voxels, the predicted moduli tend to the GW limit, but still show relatively big deviation from it. The mismatch between GW theory and numerical results for the images with relatively small pore fluid interface area, could be due to the fact that, the FEM algorithm treats the fluids in such cases as segregated phases violating the "effective fluid" assumption.

Figure 8.

To further investigate this numerical effect we increase the contact area between the pore fluids by increasing the total number of pore channels and varying the individual channel size. Specifically, we carry out simulations for three different models of the same porosity and water saturation, but with variable numbers of channels: 2166, 600 and 150 channels with each channel having cross-sectional area of 4, 16 and 64 voxels, respectively. An example of the model with 600 channels is shown in Fig. 8. The corresponding FEM simulations are shown in Fig. 9. The models with 600 and 150 channels have exactly the same total area of fluid interface. Despite this, the results are in better agreement with the GW for the 150 channel model having wider channels. At the same time, the substantially increased number of channels and, as a result, the increased area of the fluid interface for the model with 4 voxel cross-sectional area considerably improves the numerical estimates (see Fig. 7 (a) for a comparison). However, the deviation from the GW bound is still significant, and the pressure varies significantly over the pore space.

Figure 9. 
The results demonstrate that in the numerically simulated process of pressure equilibration, both the total area of the water-gas interface in pore connecting channels and channel size play a key role. That is, for a given area of the water-gas interface, the size of the individual channels needs to be relatively large ( $>4$ voxels in diameter) to ensure adequate pressure relaxation.

\subsection{Effect of spatial resolution}

Our results described above suggest that in order to obtain reliable numerical estimates of the effective elastic parameters we need to control the total area of the fluid-fluid interface and the channel size (in voxels). When dealing with actual images or synthetic models of real materials with complex morphology, it is realistic to control the size of pore interconnections only by varying the spatial resolution of the digital models.

Figure 10.

In order to verify the effect of spatial resolution, we extend the models with 2166 and 600 channels to the size of $400 \times 400 \times 400$ by doubling the number of individual elements. Fig. 10 shows that the higher-resolution numerical estimates calculated for the 2166 channel model provide better agreement with the GW theory. The output of the fluid pressure slices demonstrates better pressure equilibration for this case. However, the increased spatial resolution for the model with 600 channels does not provide an expected improvement. Instead, the results become worse, which is reflected in significant pressure variation over the pore space. We find this unexpected result to be caused by the insufficient magnitude of the tolerance parameter (relative error) used in the FEM code for this case. The tolerance on the relative changes of all stress and strain components is the stopping criterion in the process of energy relaxation. 
Figure 11.

To investigate this effect, the tolerance was changed from $10^{-4}$ to $10^{-5}$, this drastically increased the number of iterations and the time of computations, when the effective elastic properties were calculated for the 600 channel model. Fig. 11 shows a significant improvement in the equilibration of the pressure field, this improves estimates of the effective bulk moduli, which now comply very well with the GW limit. At the same time, computations for 200x200x200 models with a tolerance parameter lower than $10^{-4}$ fail to converge. We found that the optimal tolerance parameter depends on the size and geometry of the model, and the smallest admissible tolerance parameter for $200 \times 200 \times 200$ models is $10^{-4}$. Thus we conclude that the results shown in Figs. 7, 9 yield the best pressure equilibration that can be achieved with this algorithm without improving the spatial resolution of the models.

\section{Conclusions}

We have used a static FEM approach to predict the linear effective elastic properties of three dimensional materials saturated with mixture of gas and water for the first time. The numerical simulations have shown that the computed effective moduli depend significantly upon pore geometry and the size of fluid patches; this can result in discrepancies between numerics and theory. This discrepancy is a numerical artefact caused by the un-equilibrated pressure field in the pore filling mixture of gas and water. We have also shown that the pressure relaxation process is dependent upon the area of an interface between pore fluids as well as size of pore channels (in voxels). That is, for a given area of the water-gas interface, the size of the individual channels needs to be relatively large ( $>4$ voxels in diameter) to ensure pressure equilibration. 
The increased spatial resolution of digital images generally improves the numerical results. On the other hand, the numerical simulations are computationally expensive, and the number of voxels determines memory requirements and the CPU time. It is important to note that the tolerance parameter should be chosen with care as it can significantly affect the results and speed of computations.

The results of the numerical simulations confirm that the FEM code is capable of predicting the linear effective elastic properties of partially saturated rocks. However, the accuracy of the results depends on how well the digital models of materials are resolved. Employing FEM schemes with variable grid might be a solution to this problem.

\section{Acknowledgments}

This work was kindly supported by the Centre of Excellence for Exploration and Production Geophysics, CSIRO Division of Petroleum Resources, Curtin Reservoir Geophysics Consortium, CSIRO Postdoctoral Fellowship Program, Australian Research Council, and the Australian Partnership for Advanced Computing.

\section{References}

Adler, P., Jacquin, C., Thovert, J.-F., 1992. The formation factor of reconstructed porous media. Water Resources Research 28, 1571.

Arns, C. H., Knackstedt, M. A., Pinczewski, W. V., Garboczi, E. J., 2001. Accurate estimation of transport properties from microtomographic images. Geophysical Research Letters 28, 33613364. 
Arns, C. H., 2002. The influence of morphology on physical properties of reservoir rocks. Ph. D. Dissertation, University of New South Wales, Sydney, Australia, http://wwwrsphysse.anu.edu.au/ cha110/thesis/thesis.php, [accessed 13 December 2007],244 pp.

Arns, C. H., Knackstedt, M. A., Pincewski, W. V., Garboczi, E. J., 2002. Computation of linear elastic properties from microtomographic images: Methodology and agreement between theory and experiment. Geophysics 67, 1396-1405.

Arns, C. H., Knackstedt, M. A., Pinczewski, W. V., Martys N. S., 2004. Virtual permeametry on microtomographic images. Journal of Petroleum Science and Engineering 45, 1-2, 41-46.

Auzerais, F. M., Dunsmuir, J., Ferreol, B. B., Martys, N., Olson, J., Ramakrishnan, T. S., Rothman, D. H., Schwartz, L. M., 1996. Transport in sandstone: A study based on three dimensional microtomography. Geophysical Research Letters 23, 705-708.

Bear J., 1988. Dynamics of Fluids in Porous Media, Dover, New York, 764 pp.

Biot, M. A., 1962. Mechanics of deformation and acoustic propagation in porous media. Journal of Applied Physics 33, 1482-1498.

Dunsmuir, J. H., Ferguson, S. R., D’Amico, K. L., 1991. Design and operation of an imaging Xray detector for microtomography. Institute of Physics: Conference Series 121, 257-261.

Flannery, B. P., Deckman, H. W., Roberge, W. G., D’Amico, K. L., 1987. Three- dimensional X-ray microtomography. Science 237, 1439-1444.

Dvorkin J., Moos, D., Packwood, J. L., Nur, A. M., 1999. Identifying patchy saturation from well logs. Geophysics, 64, 1756-1759.

Fredrich, J., Menendez, B., Wong, T. F., 1995. Imaging the pore structure of geomaterials. Science 268, 276-279.

Garboczi, E. J., 1998. Finite element and finite difference programs for computing the linear electric and linear elastic properties of digital images of random materials. National institute of 
standards and technology, Internal Report 6269, USA, 202 pp. http://ciks.cbt.nist.gov/monograph

Gassmann, F., 1951. Uber die Elastizitat poroser Medien (Elasticity of porous media) Vierteljahrsschrift der Naturforschenden Gesselschaft in Zurich 96, 1-23.

Grechka, V., 2003. Effective media: A forward modeling view. Geophysics 68, 2055-2062.

Gurevich, B., Saenger, E. H., Ciz, R., 2005. Poroelastic effect on the shear wave in the systems of alternating solid and viscous fluid layers: Theory vs numerical modelling. In: Proceedings 75th SEG Conference\&Exhibition, Houston, USA, pp. 1601-1605.

Hill, R., 1961. Elastic properties of reinforced solids: some theoretical principles. Journal of the Mechanics and Physics of Solids 11, 357-372.

Johnson, D. L., 2001. Theory of frequency dependent acoustics in patchy-saturated porous media. Journal of Acoustic Society of America 110, 682-694.

Keehm, Y., Mukerji, T. Nur, A., 2004. Permeability prediction from thin sections: 3D reconstruction and lattice-boltzmann flow simulation. Geophysical Research Letters 31, L04606. doi: 10.1029/2003GL018761.

Knackstedt, M. A., Arns, C. H., Pinczewski, W. V., 2003. I. Accurate velocity model for clean consolidated sandstones. Geophysics 68(6), 1822-1834.

Knackstedt, M. A., Arns, C. H., Pinczewski, W. V., 2005. Velocity-porosity relationships: Predictive velocity model for cemented sands composed of multiple mineral phases. Geophysical Prospecting 53(3), 349-372.

Mavko, G., Mukerji, T. Dvorkin, J., 1998. The Rock Physics Handbook, Cambridge University Press, Cambridge, 339 pp.

Roberts, A. P., Garboczi, E. J., 2000. Elastic properties of model porous ceramics. Journal of the American Ceramic Society 83, 3041-3048. 
Roberts, A. P., Garboczi, E. J., 2002. Computation of the linear elastic properties of random porous materials with a wide variety of microstructure. In: Proceedings of the Royal Society of London A 458, 1033-1054.

Saenger, E. H., Krüger, O. S., Shapiro, S. A., 2004. Numerical considerations of fluid effects on wave propagation: Influence of the tortuosity. Geophysical Research Letters 31, L21613, doi: 10.1029/2004GL020970

Schwartz, L. M., Auzerais, F. M., Dunsmuir, J., Martys, N., Bentz, D. P., Torquato, S., 1994. Transport and diffusion in three-dimensional composite media. Physica A 207, 28-36.

Spanne, P., Thovert, J., Jacquin, J., Lindquist, W. B., Jones, K., Coker, D., 1994. Synchrotron computed microtomography of porous media: Topology and transports. Physical Review Letters 73, 2001-2004.

Toms, J., Muller, T., Ciz, R., Gurevich, B., 2006. Comparative review of theoretical models for elastic wave attenuation and dispersion in partially saturated rocks. Soil Dynamics and Earthquake Engineering 26, 548-565.

Wood, A. B., 1955. A Textbook of Sound, 3rd edn., G. Bell and Sons Ltd., London, 610 pp. Yeong, C. L. Y., and Torquato, S., 1998. Reconstructing random media. Physical Review E 57, 495-506. 


\section{Figure Captions}

Figure 1. Central slices of 3D Boolean models: local patchy model (a) and macro patchy model (b) consisting of three phases (quartz - grey, water - white, gas - black).

Figure 2. Comparison of numerical estimates of effective bulk moduli $(K)$ to $\mathrm{GW}$ and $\mathrm{GH}$ limits for two Boolean models: local patchy (LP) and macro patchy (MP).

Figure 3. Variation of pore fluid pressure in central slices of local patchy model (a) and macro patchy model (b).

Figure 4. Central slices of digital models with fluid constituents (no solid phase). Gas depicted in black, water in white.

Figure 5. Comparison of numerical estimates of effective bulk moduli (K) to GW limit for two models with fluid constituents (no solid phase). Centred model contains a single gas inclusion in centre of a liquid cube, and random model has randomly distributed gas inclusions within liquid.

Figure 6. Pore space image of an idealized poroelastic material with six channels. Blue volume represents gas-filled pore space, red volume represents water-filled pore space. Transparent part is rock frame.

Figure 7. Comparison of results of numerical simulations for images with different width of channels: 2 voxels (a, d), 10 voxels (b, e), and 30 voxels (c, f). Plots (a, b, c) show FEM estimates of effective bulk moduli (black circles) versus GW (solid line) and GH (dashed line) limits. Panels (d, e, f) show central slices of fluid pressure (in Pa) at end of relaxation process. 
Figure 8. Pore space image of an idealized poroelastic material with six hundred channels. Blue volume represents gas-filled pore space, red volume represents water-filled pore space. Transparent part is rock frame.

Figure 9. Comparison of numerical results for models with different number and width of channels: 2166 channels (a, d), 600 channels (b, e), 150 channels (c, f) with 4, 16, 64 voxels cross-sectional areas respectively. Plots a, b, and c show FEM estimates of effective bulk moduli (K) (black circles) versus GW (solid line) and GH (dashed line) limits. Panels (d, e, f) show central slices of fluid pressure (in $\mathrm{Pa}$ ) at end of relaxation process.

Figure 10. Comparison of numerical results for images with increased spatial resolution (b, f) and (d, h) to original models (a, e) and (c, g) respectively. Panels (a, e), (b, f) show results for the 2166 channel model and panels (c, g), (d, h) for the 600 channel model. Plots (a, b, c, d) show FEM estimates of effective bulk moduli (K) (black circles) versus GW (solid line) and GH (dashed line) limits. Panels (e, f, g, h) show central slices of fluid pressure (in Pa) at end of relaxation process.

Figure 11. Comparison of numerically obtained results for the 600 channel model with increased spatial resolution (b, d) to original model (a, c). The computations for upscaled model were done using the tolerance parameter of $10^{-5}$. Plots $(\mathrm{a}, \mathrm{b})$ show FEM estimates of effective bulk moduli (K) (black circles) versus GW (solid line) and GH (dashed line) limits. Panels (c, d) show central slices of fluid pressure (in $\mathrm{Pa}$ ) at end of relaxation process. 

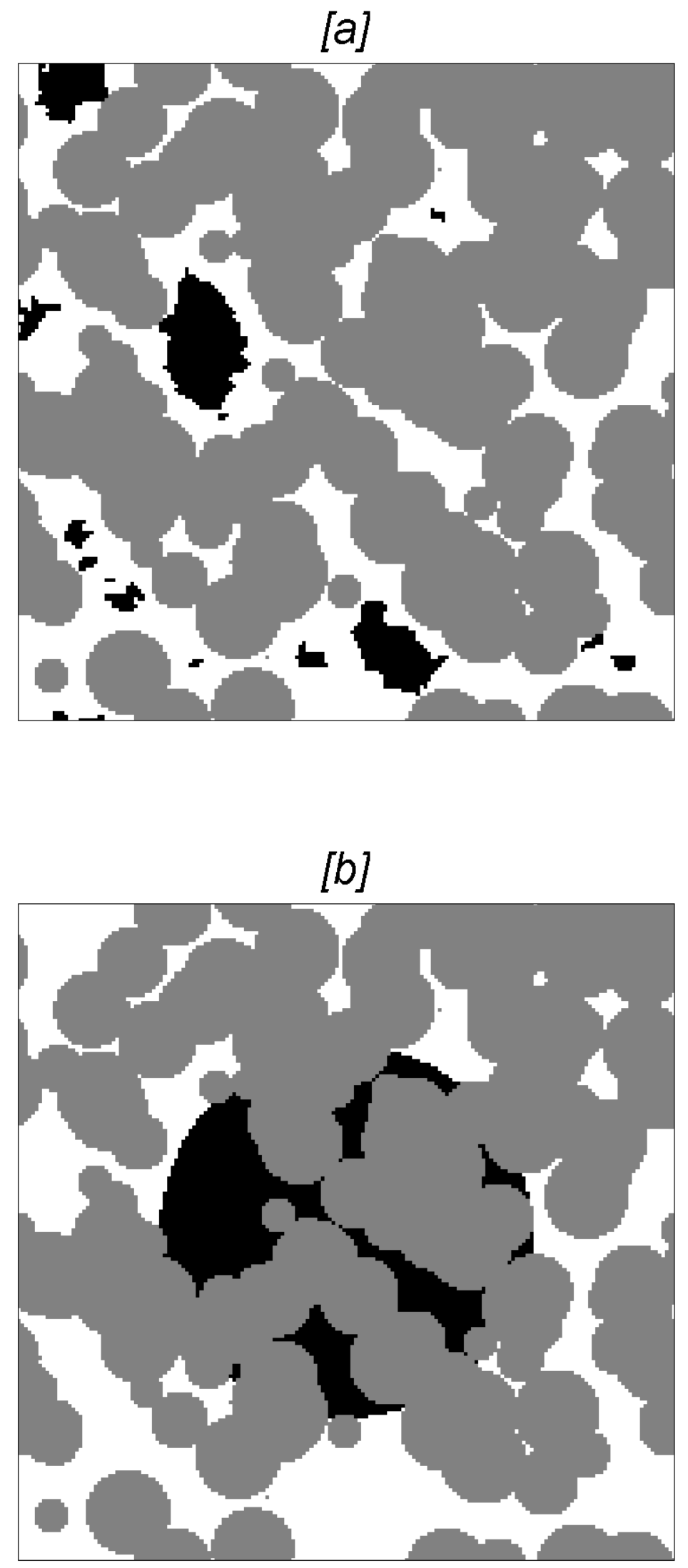

Figure 1. Central slices of 3D Boolean models: local patchy model (a) and macro patchy model (b) consisting of three phases (quartz - grey, water - white, gas - black). 


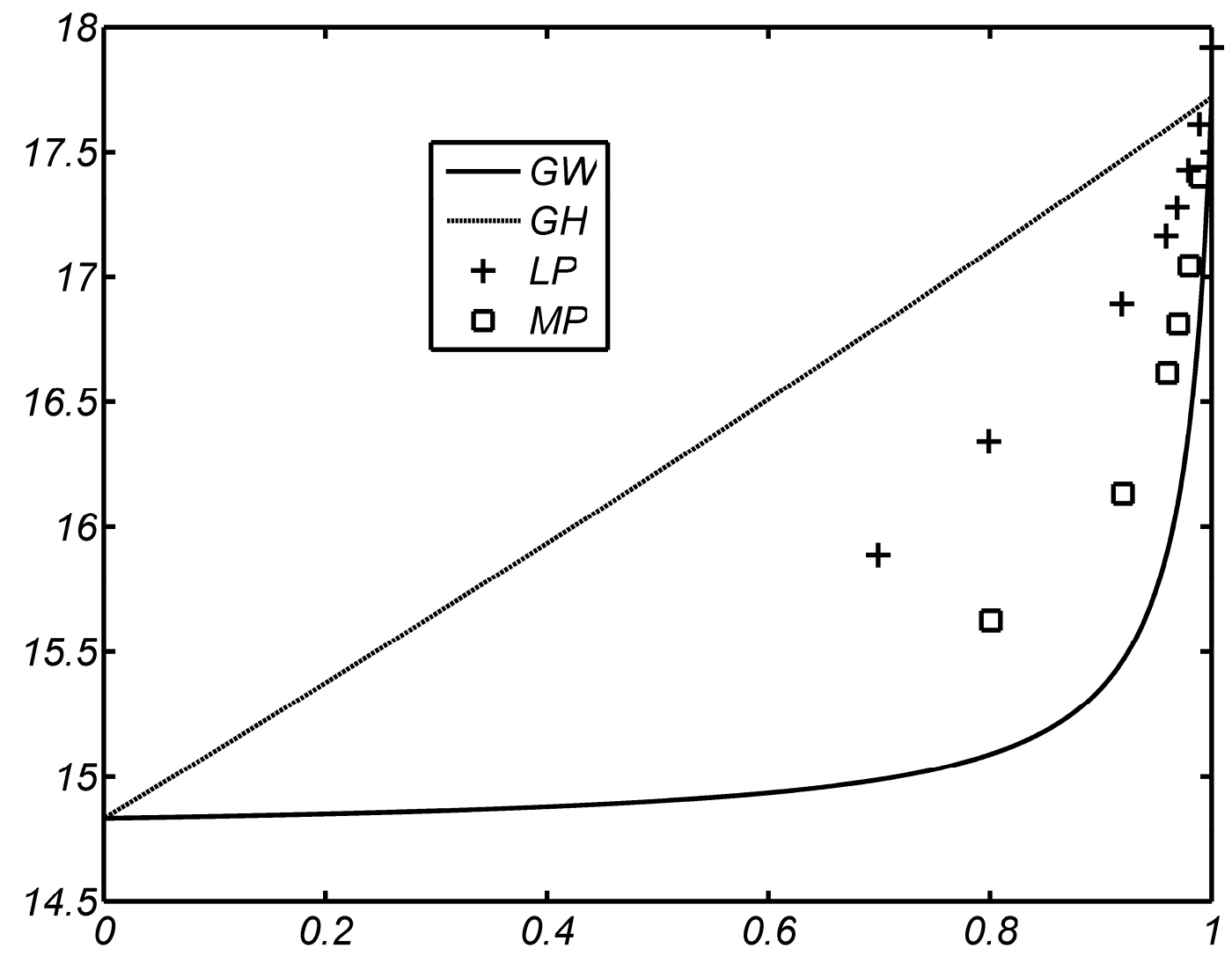

Figure 2. Comparison of numerical estimates of effective bulk moduli (K) to GW and GH limits for two Boolean models: local patchy (LP) and macro patchy (MP). 

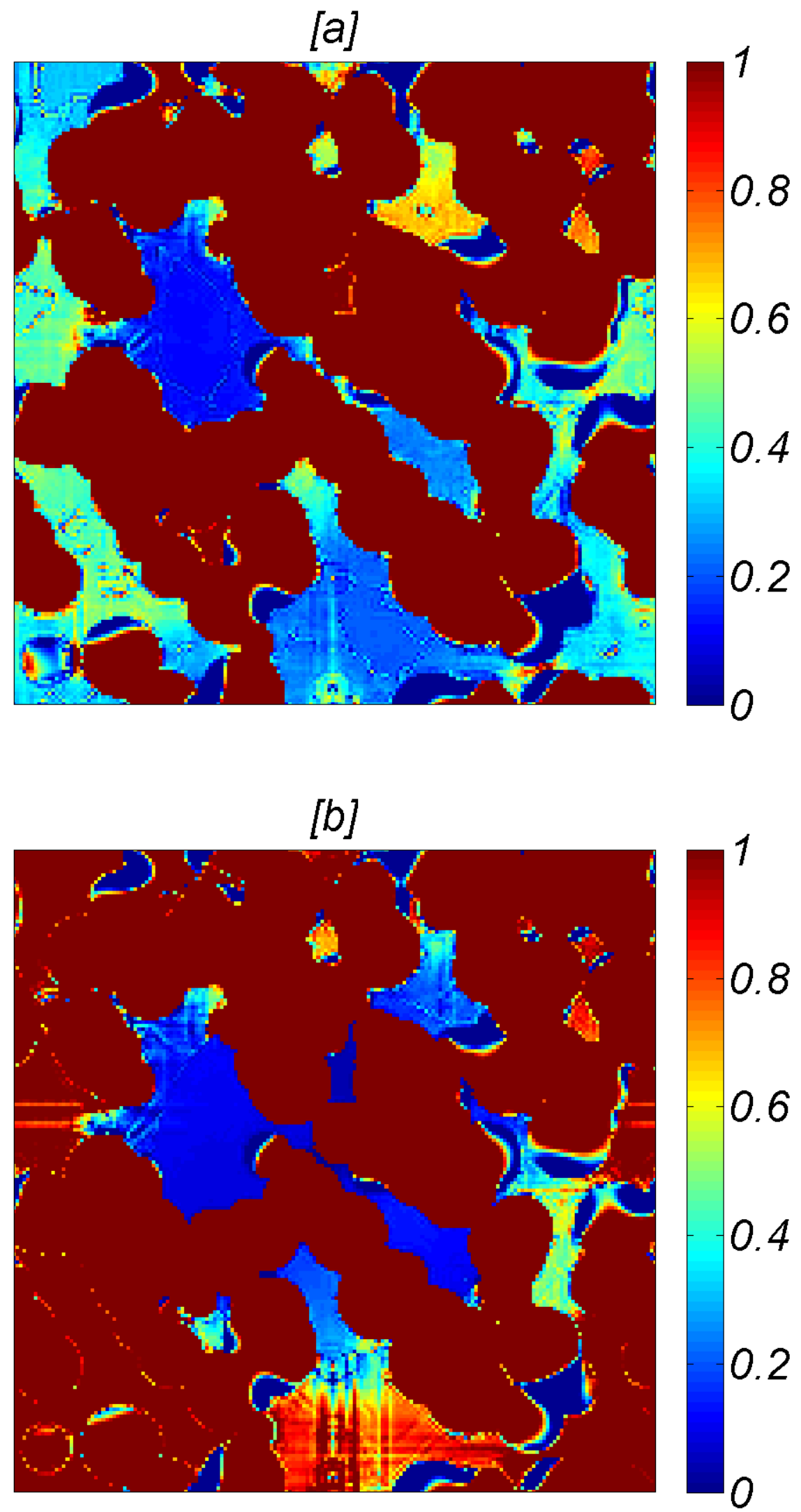

Figure 3. Variation of pore fluid pressure in central slices of local patchy model (a) and macro patchy model (b). 
[a]

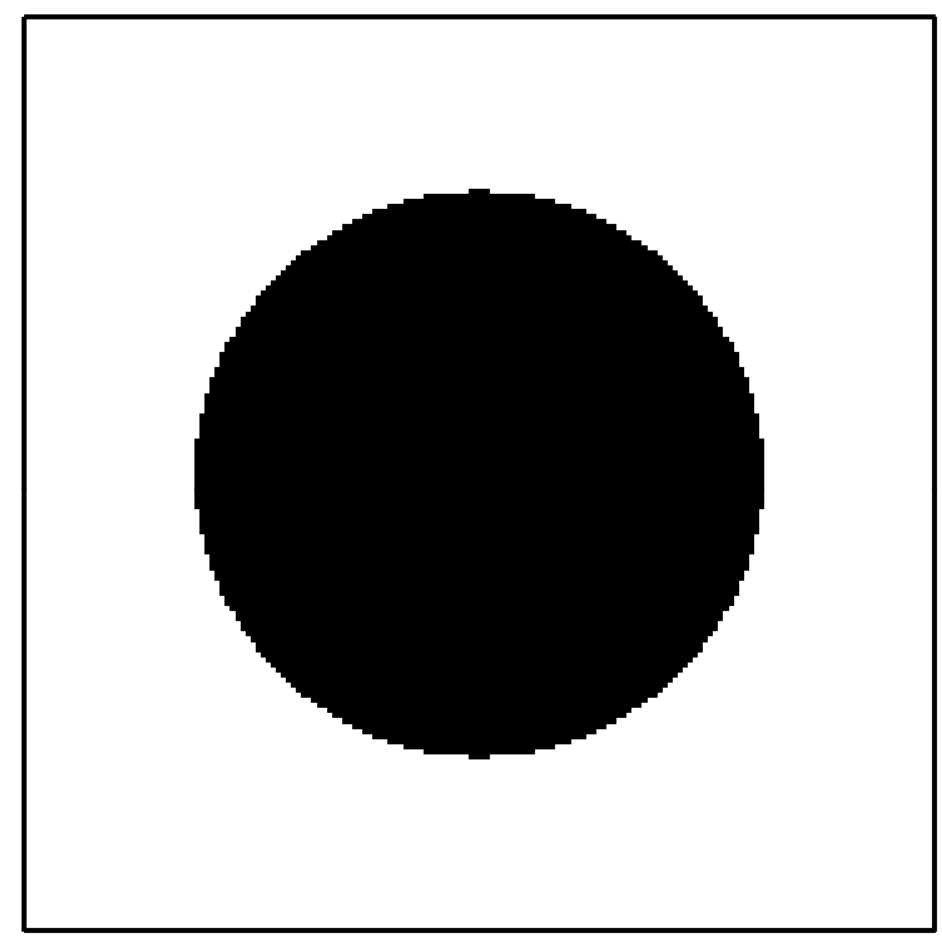

[b]

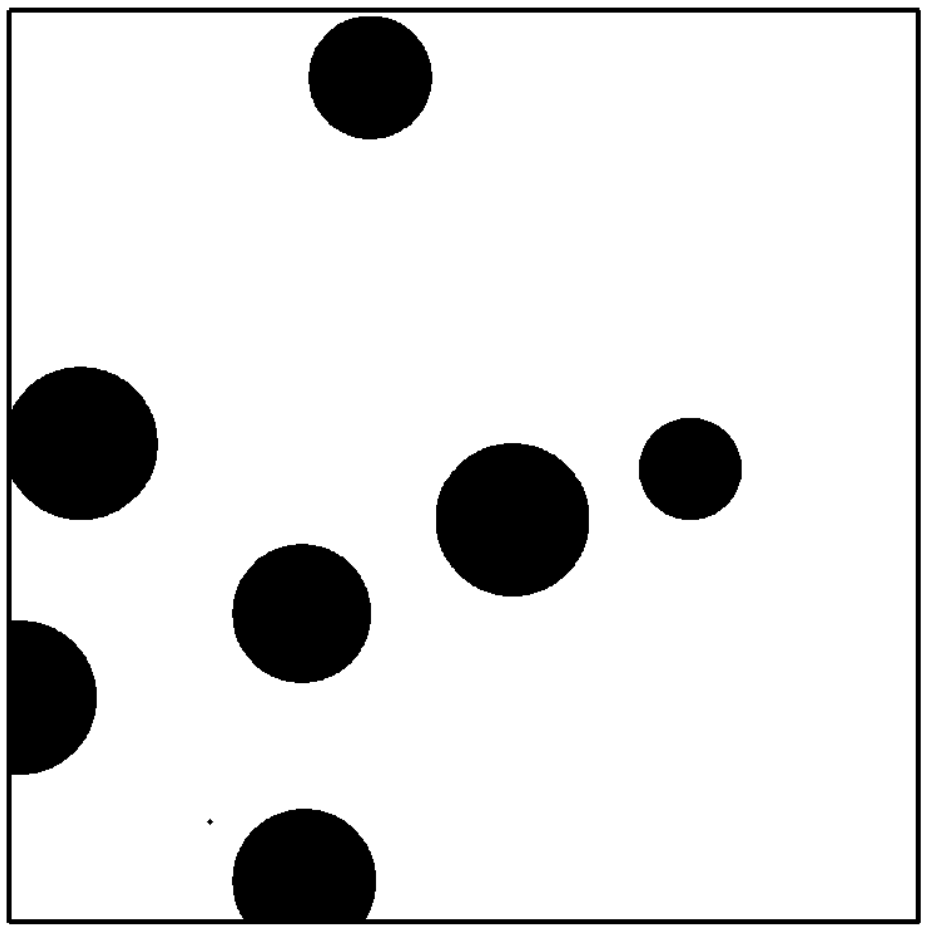

Figure 4. Central slices of digital models with fluid constituents (no solid phase). Gas depicted in black, water in white. 


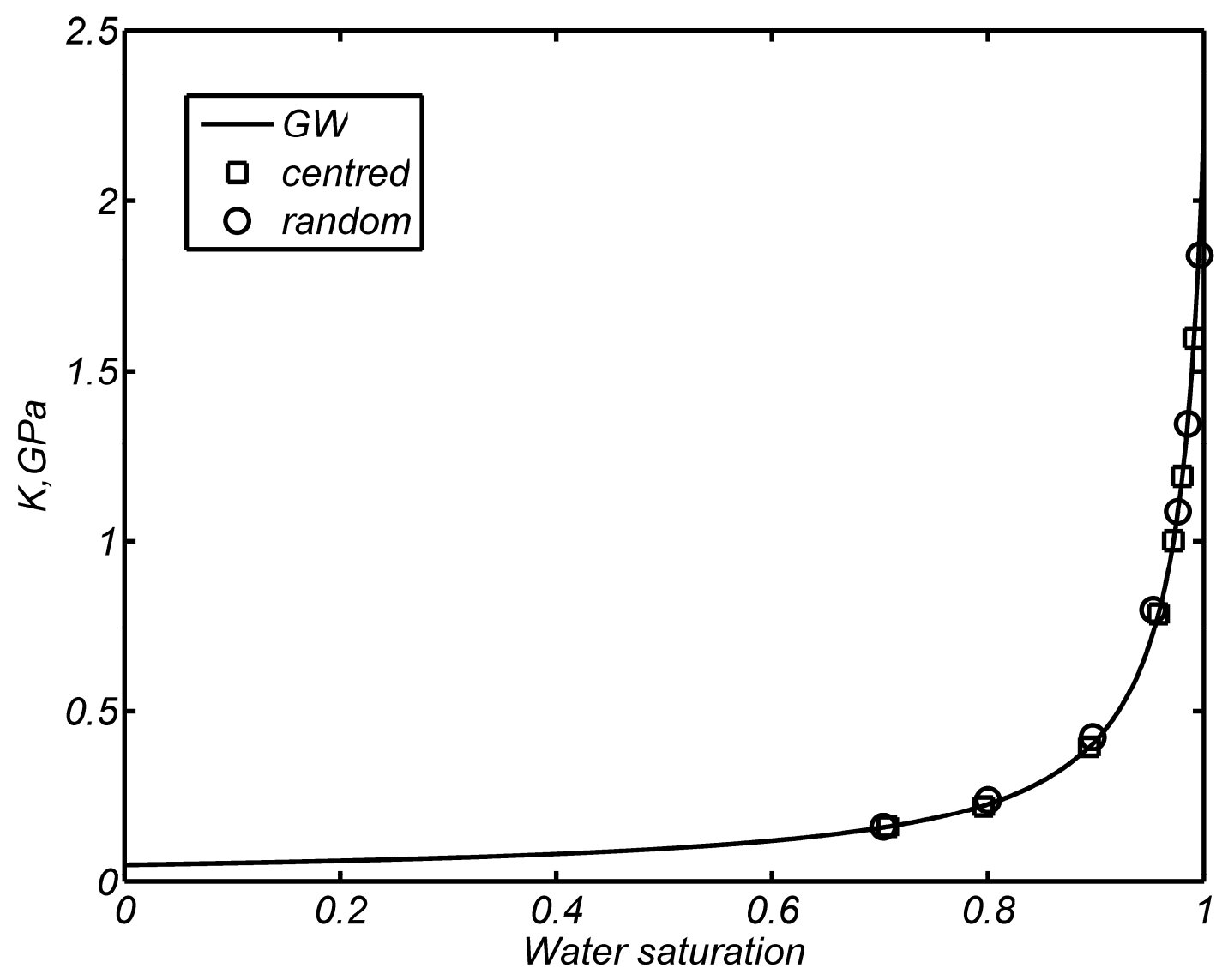

Figure 5. Comparison of numerical estimates of effective bulk moduli (K) to GW limit for two models with fluid constituents (no solid phase). Centred model contains a single gas inclusion in centre of a liquid cube, and random model has randomly distributed gas inclusions within liquid. 


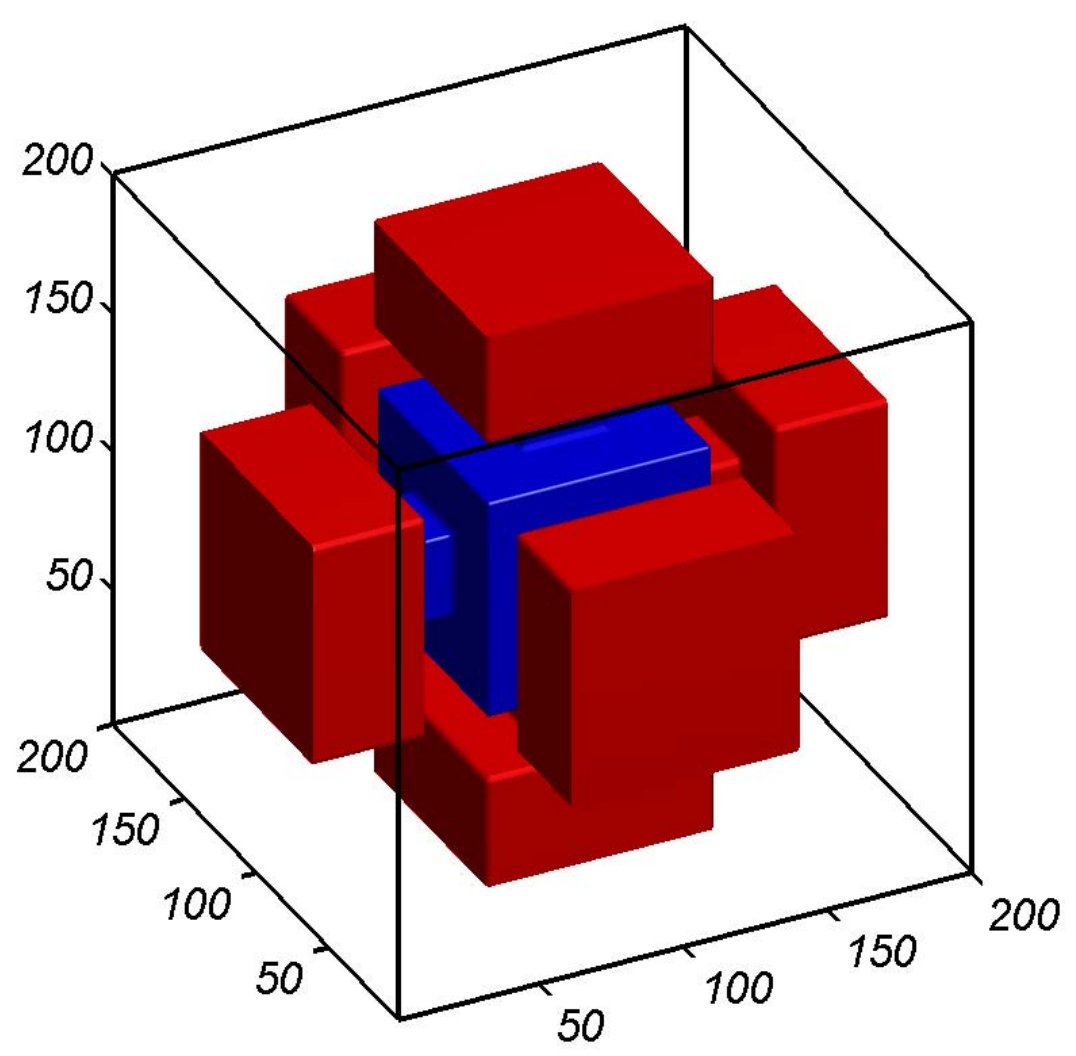

Figure 6. Pore space image of an idealized poroelastic material with six channels. Blue volume represents gas-filled pore space, red volume represents water-filled pore space. Transparent part is rock frame. 

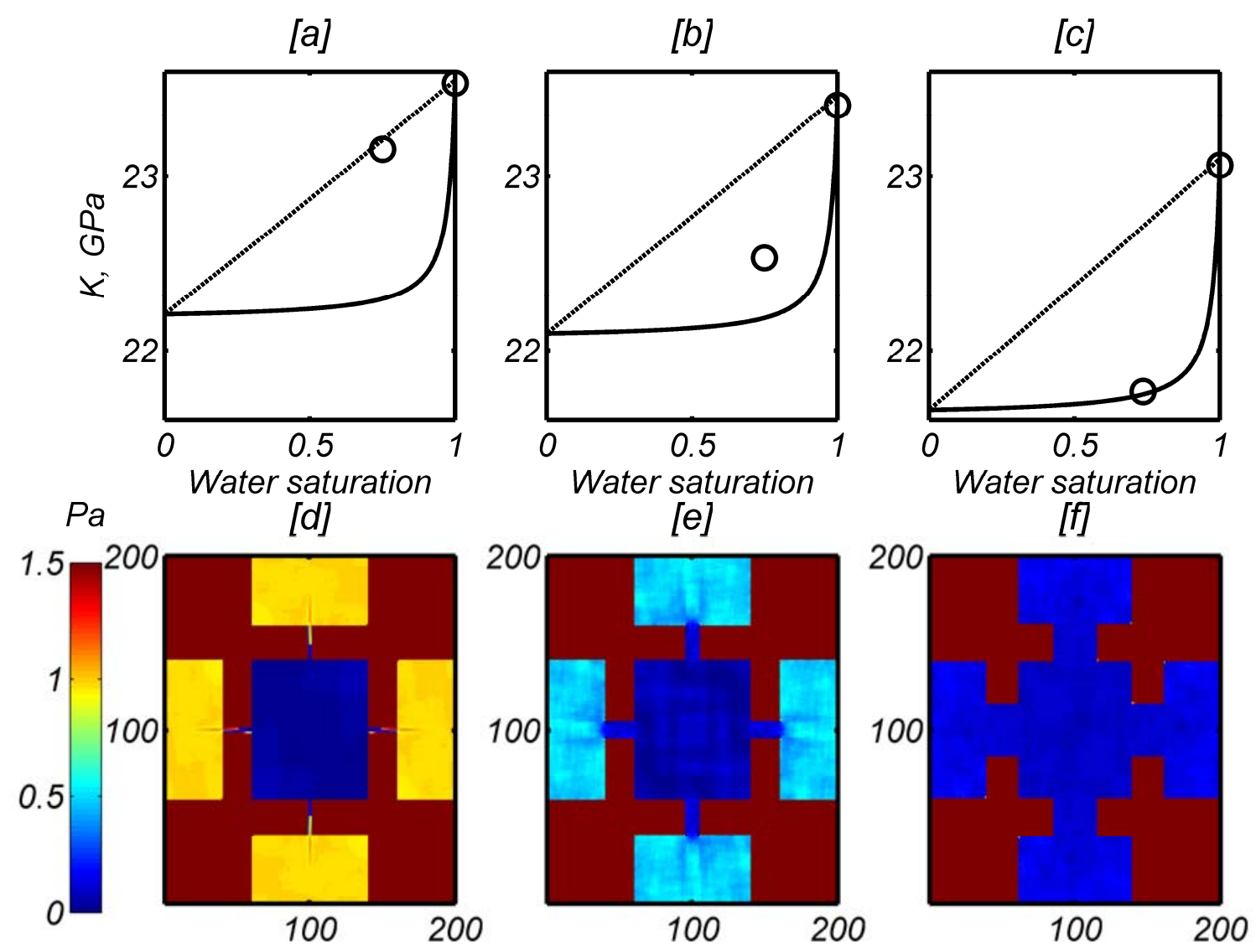

Figure 7. Comparison of results of numerical simulations for images with different width of channels: 2 voxels (a, d), 10 voxels (b, e), and 30 voxels (c, f). Plots (a, b, c) show FEM estimates of effective bulk moduli (black circles) versus GW (solid line) and GH (dashed line) limits. Panels (d, e, f) show central slices of fluid pressure (in Pa) at end of relaxation process. 


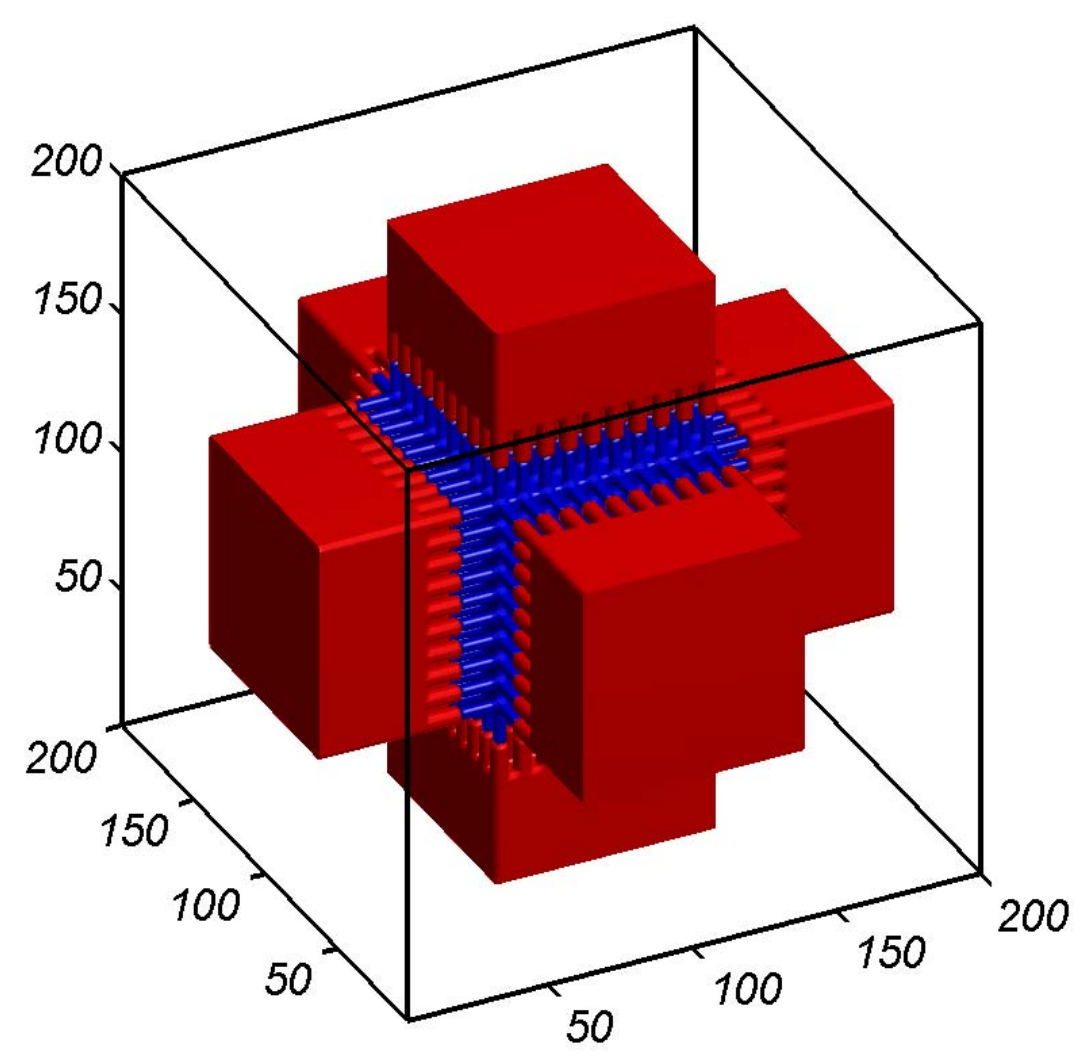

Figure 8. Pore space image of an idealized poroelastic material with six hundred channels. Blue volume represents gas-filled pore space, red volume represents water-filled pore space. Transparent part is rock frame. 

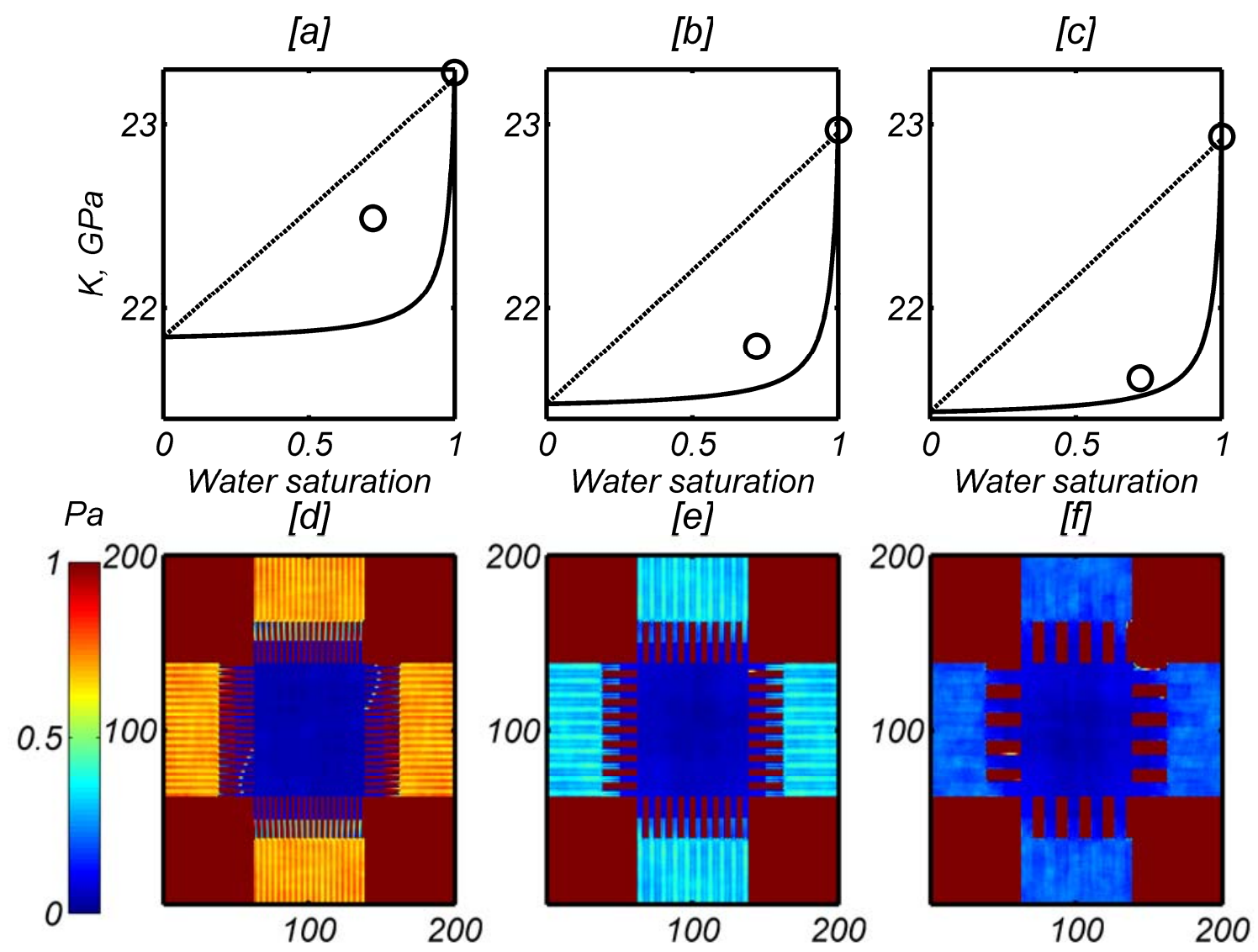

Figure 9. Comparison of numerical results for models with different number and width of channels: 2166 channels (a, d), 600 channels (b, e), 150 channels (c, f) with 4, 16, 64 voxels cross-sectional areas respectively. Plots a, b, and c show FEM estimates of effective bulk moduli (K) (black circles) versus GW (solid line) and GH (dashed line) limits. Panels (d, e, f) show central slices of fluid pressure (in $\mathrm{Pa}$ ) at end of relaxation process. 

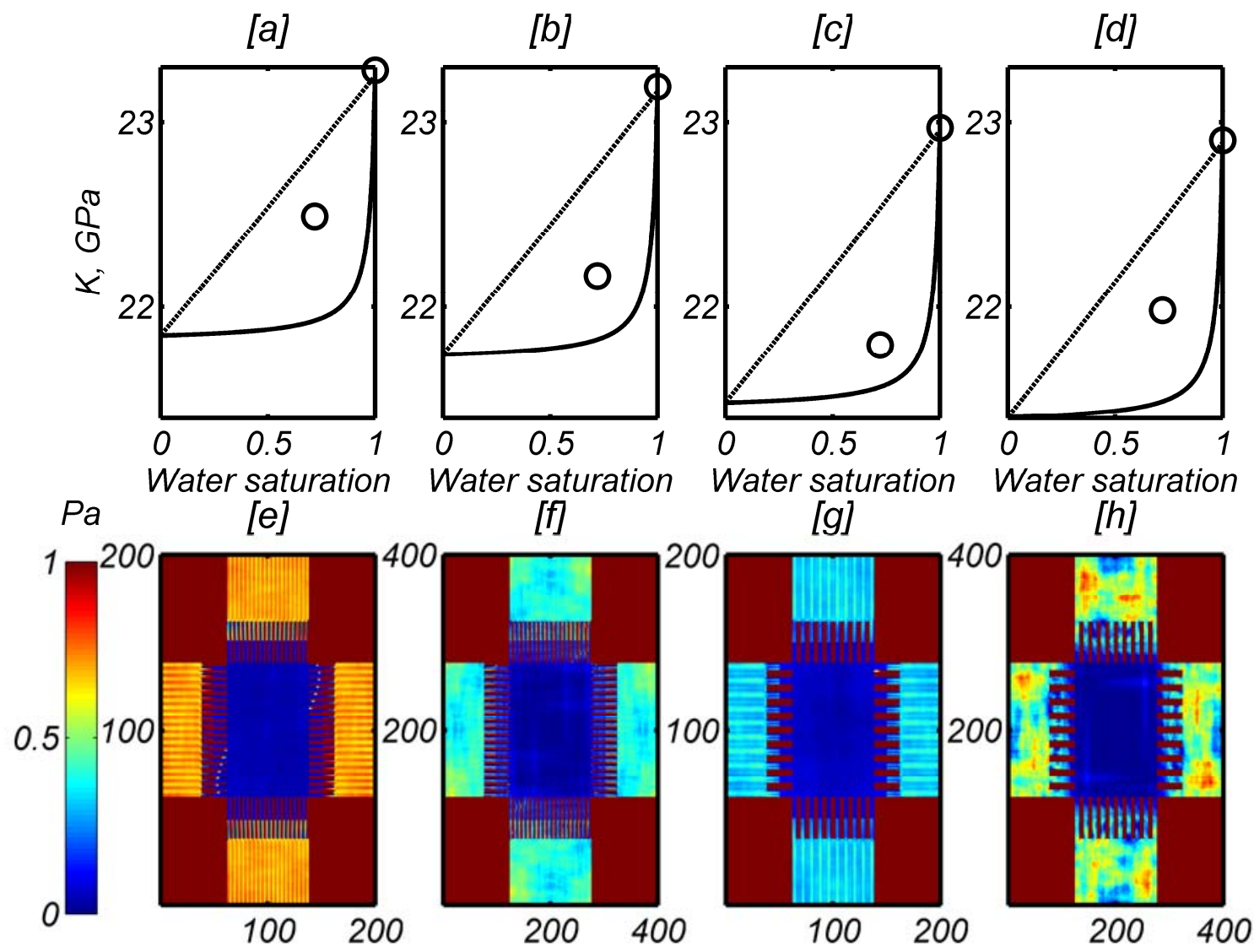

Figure 10. Comparison of numerical results for images with increased spatial resolution (b, f) and (d, h) to original models (a, e) and (c, g) respectively. Panels (a, e), (b, f) show results for the 2166 channel model and panels (c, g), (d, h) for the 600 channel model. Plots (a, b, c, d) show FEM estimates of effective bulk moduli (K) (black circles) versus GW (solid line) and GH (dashed line) limits. Panels (e, f, g, h) show central slices of fluid pressure (in Pa) at end of relaxation process. 
[a]

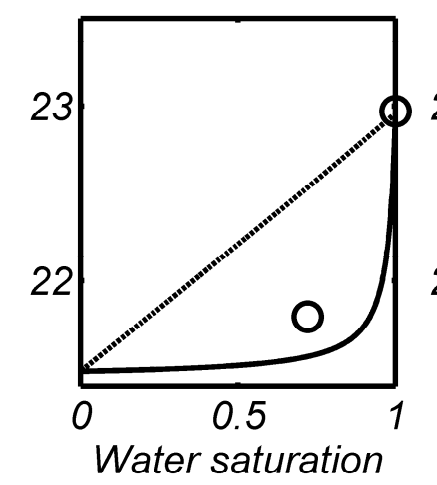

[b]

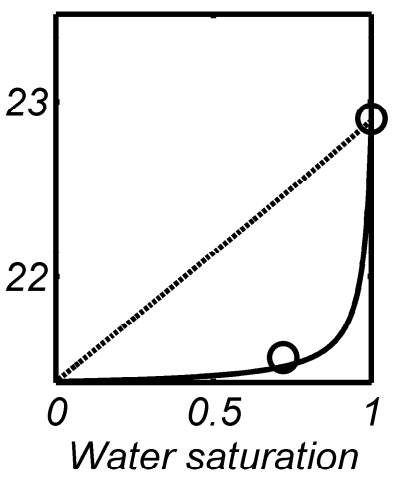

[d]

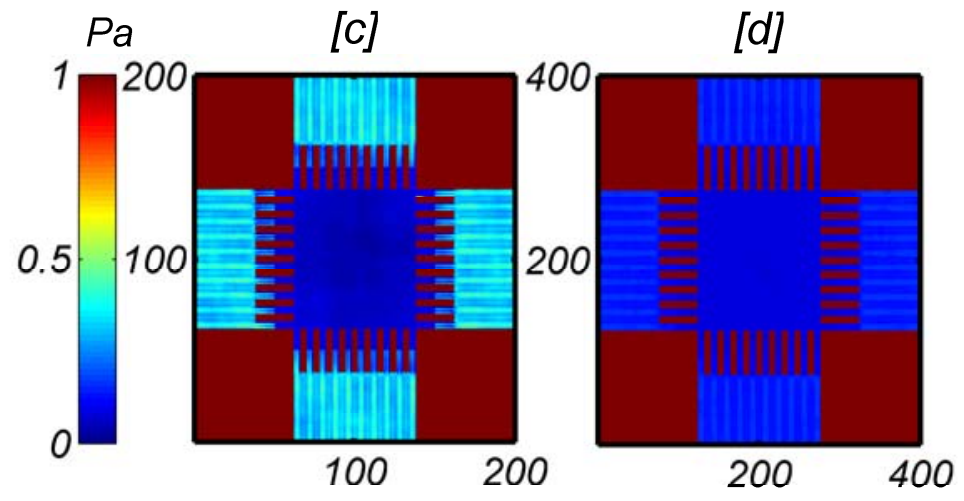

Figure 11. Comparison of numerically obtained results for the 600 channel model with increased spatial resolution (b, d) to original model (a, c). The computations for upscaled model were done using the tolerance parameter of $10^{-5}$. Plots $(\mathrm{a}, \mathrm{b})$ show FEM estimates of effective bulk moduli (K) (black circles) versus GW (solid line) and GH (dashed line) limits. Panels (c, d) show central slices of fluid pressure (in $\mathrm{Pa}$ ) at end of relaxation process. 